\title{
The Unmet Medical Need in Homozygous Familial Hypercholesterolemia
}

\author{
Dennis I Goldberg* \\ *Corresponding author: Dennis I Goldberg, PhD, FAHA, LipimetiX Development, Inc, 5 Commonwealth Rd, Suite 2A, Natick, MA 01776, USA \\ Submission: 漈 September 25, 2017; Published: 監 October 20, 2017
}

\section{Introduction}

\section{Overview of Homozygous Familial Hypercholesterolemia (HoFH)}

$\mathrm{HoFH}$ is a rare and life-threatening disease originally characterized by plasma cholesterol levels $>500 \mathrm{mg} / \mathrm{dl}$, extensive xanthomas, and marked premature and progressive atherosclerotic cardiovascular disease [1]. Genetic defects in the LDL receptor causing the inability to internalize LDL was first identified by Brown and Goldstein [2]. There are two types of familial hypercholesterolemia: the heterozygous and homozygous forms. The heterozygous form, in which the patient has one normal allele and one mutated allele, the most common form, is considered to have prevalence as high as of 1 out 2003. The homozygous form, in which the patient has two mutated alleles, has a prevalence as high as 1 in $160,0003$.

Beginning with the fetus, sustained exposure of the arterial wall to elevated LDL-C levels accelerates cholesterol deposition and vascular inflammation, resulting in the development of atherosclerosis, especially in the coronary arteries and aorta, and premature CHD $[4,5]$. Patients with homozygous FH are often diagnosed early in childhood, do not respond well to medical therapy, and can progress rapidly to premature coronary artery disease [6]. Early cardiovascular symptoms and signs are typically related to aortic stenos is and regurgitation due to massive accumulation of cholesterol in the valvular and supravalvular regions of the aortic valve, and coronary ostial stenosis [7]. Untreated, most patients with markedly elevated LDL-C levels develop overt atherosclerosis before the age of 20 years, and generally do not survive past 30 years [1]. Unfortunately, FH remains under diagnosed and undertreated despite widespread cholesterol screening [8].

\section{Genetics of HoFH}

The most common genetic defect leading to $\mathrm{FH}$ is loss-offunction mutations in the low-density lipoprotein receptor (LDLR) alleles, with more than 1600 mutations having been identified [9]. True homozygous FH, with the same mutation in both alleles, is less common than compound heterozygous $\mathrm{FH}$, with different mutations in each allele of the same gene, or double heterozygous $\mathrm{FH}$, with mutations in two different genes affecting LDL receptor function [1].
Less commonly, about 5-10\% of individuals with an FH phenotype are found to carry mutations in the ligand-binding domain of apolipoprotes in B (ApoB, 2-5\%), the protein component of the LDL particle that interacts with the LDLR. Gain-of-function mutations in the production of proprotein convertase subtilisin/kexin type 9 serine protease (PCSK9) also account for a small percentage of FH patients. $(<1 \%)$ as do loss-of-function mutations in the low-density lipoprotein receptor Adaptor Protein 1 (LDLRAP1, <1\%) [10-12]. All of these abnormalities result in a reduced ability to clear LDL-C from the circulation increased LDL-C from birth, and the above noted early cardiovascular disease [1].

\section{Need for treatment in $\mathrm{HoFH}$ patients}

Table 1: Recommended LDL cholesterol targets in $\mathrm{FH}$ guidelines [3].

\begin{tabular}{|c|c|c|}
\hline \multirow{2}{*}{ Patient } & \multicolumn{2}{|c|}{ Cholesterol Target } \\
\cline { 2 - 3 } & $\mathbf{m g} / \mathbf{d L}$ & $\mathbf{m m o l} / \mathbf{L}$ \\
\hline Children & 135 & 3.5 \\
\hline Adults & 100 & 2.5 \\
\hline $\begin{array}{c}\text { Adults with CHD or } \\
\text { Diabetes }\end{array}$ & 70 & 1.8 \\
\hline
\end{tabular}

The primary goals of management are prevention of atherosclerotic coronary artery disease by early and comprehensive controlofhypercholesterolemia and earlydetection of complications, with specific focus on ostial occlusion and aortic stenosis. Substantial improvements in the treatment of familial hypercholesterolemia have been achieved over the last 25 years with statins, ezetimibe, more potentbile acidsequestrants, VLDL synthesis inhibitors and PCSK9 antibodies. Unfortunately, many HoFH patients still do not achieve targeted LDL-C levels. [1] Randomized placebocontrolled trials to assess clinical outcomes, specifically in familial hypercholesterolemia, have not been done because of the ethical considerations of denying effective LDL-Cowering drugs to these very-high-risk patients. Still, long-term observational data from registries and specialized lipid centers demonstrate that aggressive LDL-C reduction in familial hypercholesterolemia, including the homozygous form, is associated with improved, although still high, rates of cardiovascular disease. Cholesterol reduction achieved with statin therapy, furthermore, is associated with delayed cardiovascular events and prolonged survival in patients with 
homozygous familial hypercholesterolemia [13]. Nonetheless, it is now apparent that the current LDL cholesterol guidelines for HoFH are not optimal for high risk patients [14], and even those LDL cholesterol levels remain difficult to achieve in $\mathrm{HoFH}$ with available pharmacotherapy [1] (Table 1).

Given the unmet need for further LDL-C lowering in this patient population, several newer non-statin therapeutics recently have been approved for the treatment of HoFH. While these new strategies all report significant lowering of LDL-C, each raises questions regarding utility and the risk/benefit profile.

\section{Two recently approved drugs, Kynamro $($ mipomersen), and Juxtapid $\circledast$ (lomitapide), inhibit the synthesis and secretion of VLDLin the liver:}

a) Kynamro® a second generation antisense oligonucleotide, prevents the synthesis of apolipoprotein B-100 in the liver, thereby inhibiting the formation and secretion of apolipoprotein B lipoproteins by the liver. Studies have shown that Kynamro treatment results in a sustained 25\% reduction in serum cholesterol levels. Given the risk/benefit profile of Kynamro, however, its role in treatment of patients in this population may be limited to only those with a very high risk. Of particular concern is the observation of the side effect of fatty liver; larger and longer studies may be required to determine if this is a relatively benign and reversible side effect or something more serious $[15,16]$.

b) Juxtapid $\AA$ is a small molecule microsomal triglyceride transfer protein inhibitor (MTP-I) approved as a daily oral therapeutic for the FH population. Inhibition of MTP lowers LDL-C by inhibiting apoB lipidation and reducing apoB containing lipoprotein secretion. Juxtapid, given as monotherapy or in combination with ezetimibe, lowered LDL cholesterol and apoB levels by $\sim 50 \%$ in patients with familial hypercholesterolemia (FH) or moderate hypercholesterolemia, but the clinical utility of MTP inhibitors may be restricted by their potential to induce elevation of liver enzymes and hepatic steatosis and patient toleration due to GI side effects [15].

Most recently two antibodies that inhibit PCSK9 have demonstrated efficacy by increasing residual LDL receptor activity. Significant and substantial reductions in LDL-C have been observed following subcutaneous injections of a monoclonal antibody to PCSK9 to patients with severe heterozygous FH, including those with coronary artery disease and baseline LDL-C levels over $100 \mathrm{mg} / \mathrm{dL}$ on high dose statin with or without ezetimibe [16]. The mechanism of action for PCSK9 inhibitors, however, requires the patient to have a functional LDL receptor pathway, without loss-of-function mutations in either the receptor or the LDL binding domain. The recent TESLA-B study demonstrated that Repatha ${ }^{\circledR}$ (evolocumab) reduced LDL-C from $355 \mathrm{mg} / \mathrm{dl}$ to $270 \mathrm{mg} / \mathrm{dl}$ [16] in HoFH patients already on optimal statin and ezetimibe therapy. As a result, Repatha has been approved by the FDA as adjunctive treatment in HoFH. Despite these new drug approvals, LDL-apheresis, every 1-2 weeks, remains an important option for patients with HoFH The importance of apheresis in HoFH treatment is illustrated in Figure 1, below. In this patient population, the rate of future cardiovascular events appears to be reduced upon profound lowering of LDL-C concentrations with LDL apheresis [17]. Because of the early development of atherosclerosis and premature CVD, the Consensus Panel on Familial Hypercholesterolemia of the European Atherosclerosis Society, recommends that children with HoFH should start LDL apheresis by age 5 and not later than age 8 [1]. (Figure 1)

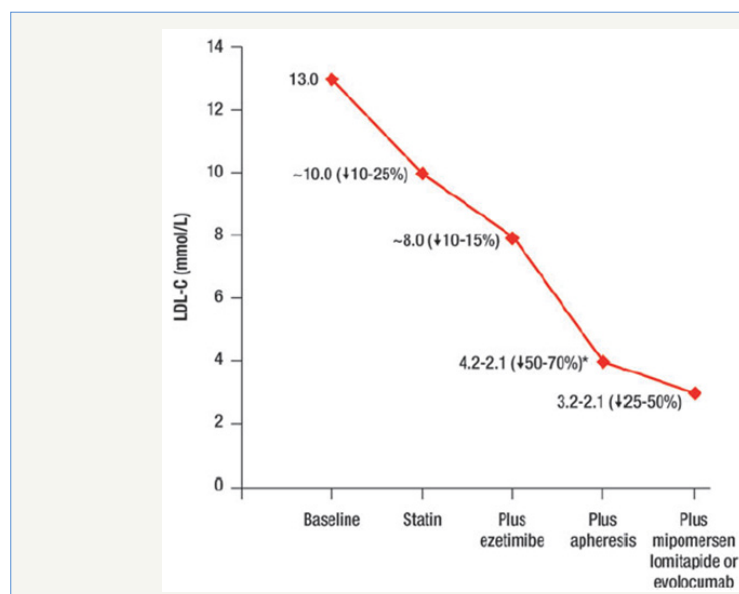

Figure 1: Cumulative LDL cholesterol lowering effects of statin, ezetimibe, adjunctive mipomersen, lomitapide or evolocumab, plus LDL-apheresis [1].

Unfortunately, LDL-apheresis is an invasive, time-consuming, and expensive intervention that is not universally available or affordable. LDL apheresis is particularly challenging for children, who often require venous access ports [18]. As a result, an unmet medical need for additional, effective, accessible and better tolerated LDL-C lowering therapies remains for many patients with HoFH [19].

\section{Conclusion}

Despite the recent approvals of new therapies, the ability to decrease LDL-C in HoFH patients remains difficult. Newer, more potent and better tolerated LDL lowering therapies are needed to prevent the atherosclerotic cardiovascular disease in these genetically challenged patients.

\section{References}

1. Cuchel M, Bruckert E, Ginsburg HN, Raal FJ, Santos RD, et al. (2014) Homozygous familial hypercholesterolaemia: new insights and guidance for clinicians to improve detection and clinical management. A position paper from the Consensus Panel on Familial Hypercholesterolaemia of the European Atherosclerosis Society. Eur Heart J 35(32): 2146-2157.

2. Brown MS, Goldstein JL (1986) A receptor-mediated pathway for cholesterol homeostasis. Science 232(4746): 34-47.

3. Noordestgaard BG, Chapman MJ, Humphries SE, Ginsberg HN, Masana L, et al. (2013) Familial hypercholesterolemia is underdiagnosed and undertreated in the general population: guidance for clinicians to prevent coronary heart disease: consensus statement of the European Atherosclerosis Society. Eur Heart J 34(45): 3478-3490.

4. Brown MS, Kovanen PT, Goldstein JL, Eeckels R, Vandenberghe K, et al. (1978) Prenatal diagnosis of homozygous familial FH in children and adolescents 2437 hypercholesterolaemia. Expression of a genetic receptor disease in utero. Lancet: 526-529. 
5. Buja LM, Kovanen PT, Bilheimer DW (1979) Cellular pathology of homozygous familial hypercholesterolemia. Am J Pathol 97(2): 327-357.

6. Palacio CH, Harring TR, Nguyen NTT, Goss JA, O’Mahony CA (2011) Homozygous Familial Hypercholesterolemia: Case Series and Review of the Literature. Case Rep Transplant 2011:154908.

7. Kolansky DM, Cuchel M, Clark BJ, Paridon S, McCrindle BW, et al. (2008) Longitudinal evaluation and assessmentof cardiovascular disease in patients with homozygous familial hypercholesterolemia. Am J Cardiol 102(11): 1438-1443.

8. Abul-Husn NS, Manickam K, Jones LK, Wright EA, Hartzel DN, et al. (2016) Genetic identification of familial hypercholesterolemia within a single U.S. health care system. Science 354(6319): 1550-1557.

9. Hobbs HH, Brown MS, Goldstein JL (1992) Molecular genetics of the LDL receptor gene in familial hypercholesterolemia. Hum Mutat 1(6): 445466.

10. Soutar AK, Naoumova RP (2007) Mechanisms of Disease: genetic causes of familialhypercholesterolemia. Nat Clin Pract Cardiovasc Med 4(4): 214-225.

11. Innerarity TL, Weisgraber KH, Arnold KS, Mahley RW, Krauss RM, et al. (1987) Familial defective apolipoprotein B-100: low density lipoproteins with abnormal receptor binding. Proc Natl Acad Sci USA 84(19): 69196923.

12. Abifadel M, Varret M, Rabeè JD, Allard D, Ouguerram K, et al. (2003) Mutations in PCSK9 causes autosomal dominant hypercholesterolemia. Nat Genet 34(2): 154-156.
13. Raal FJ, Pilcher GJ, Panz VR, van Deventer HE, Brice BC, et al. (2011) Reduction in mortality in subjects with homozygous familial hypercholesterolemia associated with advances in lipid-lowering therapy. Circulation 124(20): 2202-2207.

14. O’Keefe JH Jr, Cordain L, Harris WH, Moe RM, Vogel R (2004) Optimal lowdensity lipoprotein is 50 to $70 \mathrm{mg} / \mathrm{dL}$ : lower is better and physiologically normal. J Am Coll Cardiol 43(11): 2142-2146.

15. Rader DJ, Kastelein JJ (2014) Lomitapide and Mipomersen: Two Firstin-Class Drugs for Reducing Low-Density Lipoprotein Cholesterol in Patients With Homozygous Familial Hypercholesterolemia. Circulation 129(9): 1022-1032.

16. Feingold KR (2010) Does inhibition of apolipoprotein B synthesis produce foie gras? J Lip Res 51(5): 877-878.

17. Raal FJ, Honarpour N, Blom DJ, Hovingh GK, Feng Xu, et al. (2015) Inhibition of PCSK9 with evolocumab in homozygousfamilial hypercholesterolaemia (TESLA Part B): a randomised, double-blind, placebo-controlled trial. Lancet 385(9965): 341-350.

18. Bambauer R, Schiel R, Latza R (2003) Low-density Lipoprotein Apheresis: An Overview. Ther Apher Dial 7(4): 382-390.

19.Thompson GR, Barbir M, Davies D, Dobral P, Gesinde M, et al. (2010) Efficacy criteria and cholesterol targets for LDL apheresis. Atherosclerosis 208(2): 317-321. 